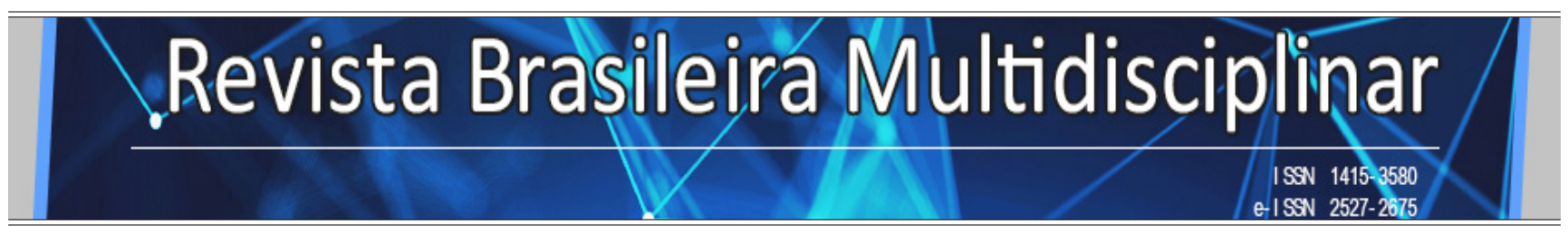

http://revistarebram.com/index.php/revistauniara

\title{
A doença de chagas no município de Abaetetuba, Pará, Brasil
}

Lanaíde Lobato Viana*; Walter Souza Santos ${ }^{\star *}$; Veracilda Ribeiro Alves ${ }^{\star *}$; Cléa Nazaré Carneiro Bichara ${ }^{\star \star *}$; Altem Nascimento Pontes ${ }^{\star \star \star \star}$

${ }^{*}$ Mestranda do programa ProfBio pela Universidade Federal do Pará.

${ }^{*}$ Instituto Evandro Chagas/SVS/MS.

***Programa de Pós-Graduação em Ciências Ambientais e Ensino em Saúde da Amazônia.

****Programa de Pós-Graduação em Ciências Ambientais - Universidade do Estado do Pará.

*Autor para correspondência e-mail: baslana@hotmail.com.

\section{Palavras-Chave}

Epidemiologia

Doenças transmitidas por Alimentos

Saúde Pública

\section{KEYWORDS}

Epidemiology

Foodborne Diseases

Public Health

\section{RESUMO}

Traçar o perfil epidemiológico da doença de Chagas aguda em Abaetetuba/ Pará, entre 2007 a 2017. Foram analisados dados secundários da Prefeitura de Abaetetuba, Secretaria de Saúde Pública do Estado do Pará e do Sistema de Informação de Agravos de Notificação, utilizando estatística descritiva. Dos 316 casos confirmados, 238 eram autóctones. A prevalência foi maior em homens (51,9\%). A incidência foi maior na faixa etária de 20 a 59 anos com ou sem nenhuma escolaridade. Dois óbitos foram notificados. O número de casos foi comparável entre a zona urbana $(52,85 \%)$ e a rural (43,67\%). A via oral foi a mais notificada (73,42\%). O critério laboratorial elucidou $97 \%$ dos casos. A incidência mensal foi maior nos meses de julho a dezembro, coincidindo com a safra do açaí. A via oral é um importante componente e a adoção de boas práticas na produção do açaí pode diminuir o número de casos da doença.

\section{Abstract}

Chagas disease in the municipality of Abaetetuba, Pará, Brazil

To trace the epidemiological profile of acute Chagas disease in Abaetetuba / Pará, between 2007 and 2017. Secondary data from the Abaetetuba City Hall, the State of Pará Public Health Secretariat and the Notifiable Diseases Information System were analyzed, using descriptive statistics. Results: Of the 316 confirmed cases, 238 were indigenous. The prevalence was higher in men (51.9\%). The incidence was higher in the 20 to 59 age group with or without any education. Two deaths were reported. The number of cases was comparable between the urban area $(52.85 \%)$ and the rural area $(43.67 \%)$. The oral route was the most reported $(73.42 \%)$. The laboratory criterion clarified $97 \%$ of the cases. The monthly incidence was higher from July to December, coinciding with the açaí harvest. The oral route is an important component and the adoption of good practices in the production of açaí can reduce the number of cases of the disease. 


\section{INTRODUÇÃo}

A doença de Chagas (DC) é uma importante doença tropical negligenciada e um dos principais problemas de saúde pública na América Latina (MONCAYO; SILVEIRA, 2009), que tem como agente etiológico o parasito Trypanosoma cruzi (CHAGAS, 1909)

A doença pode se apresentar de duas formas, a aguda (DCA) e a crônica (DCC) (MONTEIRO, et al., 2013). A primeira, menos severa, até mesmo assintomática em alguns casos, com período de incubação que pode variar de 4 a 8 dias, para o aparecimento dos primeiros sintomas; já a segunda, resultante de complicações da primeira, ocasiona um quadro clínico mais severo, que pode persistir por anos, comprometendo tecidos cardíacos e estomacais, podendo levar o indivíduo a morte súbita por insuficiência cardíaca (FERREIRA, et al., 2014)

A principal via de transmissão do T. cruzi é a vetorial, que é mediada por insetos da subfamília Triatominae que são comumente conhecidos como barbeiros, mas esta denominação pode variar dependendo da região (RODRIGUES, et al., 2016). As vias vertical e oral (na qual há participação dos vetores) são relativamente importantes, enquanto que os casos associados a transfusões sanguíneas ou transplantes de órgãos são raros atualmente; outras vias incomuns são a manipulação de animais infectados, os acidentes em laboratório e a transmissão sexual (COURA, 2008).

Dados da OMS indicam que haja entre 6 a 7 milhões de pessoas infectadas pelo T. cruzi em todo mundo (WORLD HEALTH ORGANIZATION, 2015). Atualmente, devido à migração de pessoas infectadas, a doença está também presente na Europa, Japão e Austrália (GASCON, et al, 2010), mas a maioria dos infectados está distribuída entre 21 países da América Latina.

No Brasil, estimativas recentes indicam que o número de pessoas infectadas varia entre 1,9 a 4,6 milhões (MARTINS-MELO, et al., 2014), o que corresponderia à variação de 1,0 a 2,4\% da população (DIAS, et al., 2014). Entre os anos 2000 a 2013, a incidência anual da doença foi de 0,061 casos/100.000 habitantes (BRASIL, 2015). Entre 2007 a 2016, foram confirmados 2.345 casos agudos da doença, com uma média anual de 213 casos, sendo 2.235 (95,3\%) somente na região Norte, nos estados do Amapá (6\%), Amazonas (5,32\%) e Pará (85,45\%). (SINAN/DATASUS, 2018).

O município de Abaetetuba, no estado do Pará, desde 1999, concentra aproximadamente 15\% dos casos de DCA na Amazônia. No período de 2006 a 2013, foram notificados 7.120 casos de DCA no estado do Pará, sendo 2.918 (31,7\%) só em Abaetetuba, colocando-o como primeiro na comparação entre os 10 municípios paraenses com maiores notificações da doença (SOUZA, et al., 2014).

Atualmente, a Amazônia brasileira é considerada uma grande região endêmica para a DCA, principalmente pelo grande consumo de açaí pela população e as precárias condições de higiene, principalmente nos interiores (PINTO, et al.,2008).

Dados do IBGE mostram que em 2015 o município de Abaetetuba figurou em segundo lugar na produção estadual e nacional de açaí, produzindo 165.750 toneladas do fruto (IBGE, 2010). Por um lado, esses dados são relevantes, porque a produção do açaí em larga escala tem gerado trabalho e renda para os munícipes locais, por outro, geram bastante preocupação na área da saúde pública, pois a forma de contágio mais importante da DC na região é pela ingestão do suco de açaí (MARQUES, et al., 2013).

Considerando o elevado número de casos da DCA no estado do Pará, em especial no município de Abaetetuba, onde a via oral é o principal modo de transmissão, implicado com envolvimento do consumo do suco de açaí, este estudo objetivou traçar o perfil epidemiológico da DCA no município no período de 2007 a 2017.

\section{MATERIAIS E MÉTODO ÁreA DE ESTUdo}


Abaetetuba está situado na mesorregião do Nordeste Paraense (0143’24” S e 4852'54” W). O contingente populacional de Abaetetuba em 2010 era de 141.100 habitantes, com estimativa de 153.380 habitantes para 2017. O município apresenta áreas urbanas e rurais, esta última com 72 ilhas. A população da área urbana em 2010 era de 82.998 habitantes e da rural de 58.102. O clima do município apresenta bastante umidade com altas temperaturas, sendo o solo predominante do tipo latossolo amarelo distrófico, enquanto que nas ilhas predominam as várzeas de marés com não uniformidade na topografia (SANTOS; COELHO-FERREIRA, 2012). A Figura 1 apresenta o mapa de localização do município de Abaetetuba, Pará.

Figura 1 - Mapa indicando a localização do município de Abaetetuba, Pará. Fonte: IBGE 2018.

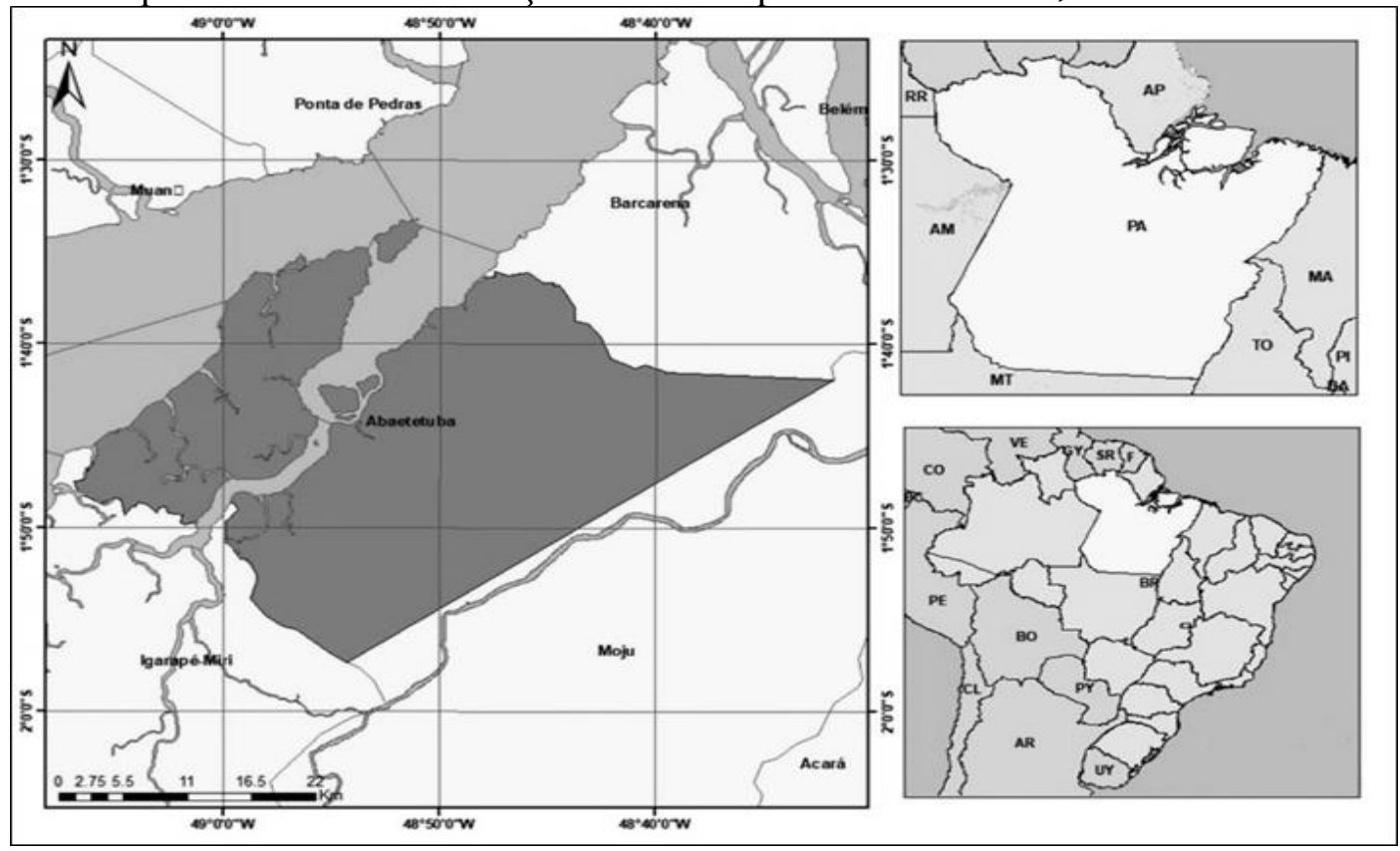

Fonte: Aquino, RDG.

\section{OBTENÇÃo E ANÁlise dos dAdos}

O método da pesquisa consistiu na análise de dados documentais dos registros sobre a DCA de 2007 a 2017, disponibilizados pelo setor de epidemias da Prefeitura de Abaetetuba e pela Secretaria de Saúde Pública do Estado do Pará (SESPA), complementados com os dados disponíveis na base de dados do Sistema de Informação de Agravos de Notificação - SINAN do Ministério da Saúde (disponível em http:// datasus.saude.gov.br/).

Com a abordagem quantitativa, os dados foram analisados utilizando estatística descritiva, com cálculos de médias e frequências, acompanhadas de Intervalos de Confiança de $95 \%\left(\mathrm{IC}_{95 \%}\right)$, e apresentação de gráficos e tabelas.

\section{RESULTADOS E DISCUSSÃo}

Entre 2007 a 2017, foram confirmados 316 casos de DCA em Abaetetuba, com média de aproximadamente 32 casos por ano. Desse total, 238 eram autóctones, dois alóctones e aproximadamente $32 \%(n=76)$ não tinham informações detalhadas sobre o município de residência (branco) do indivíduo ou foram considerados como indeterminados, denotando a incompletude dos dados epidemiológicos sobre o agravo, bem como a necessidade do preenchimento dessas informações nas fichas de notificação. 
Na comparação mensal do número de casos de DCA, observa-se um padrão em todos os anos, com um aumento significativo das notificações a partir do mês de junho até dezembro, conforme apresentado na Figura 2.

Figura 2 - Consolidado do número de casos de DCA por mês, no período de 2007 a 2017, no município de Abaetetuba, Pará. A linha tracejada representa uma linha de tendência móvel para o período analisado.

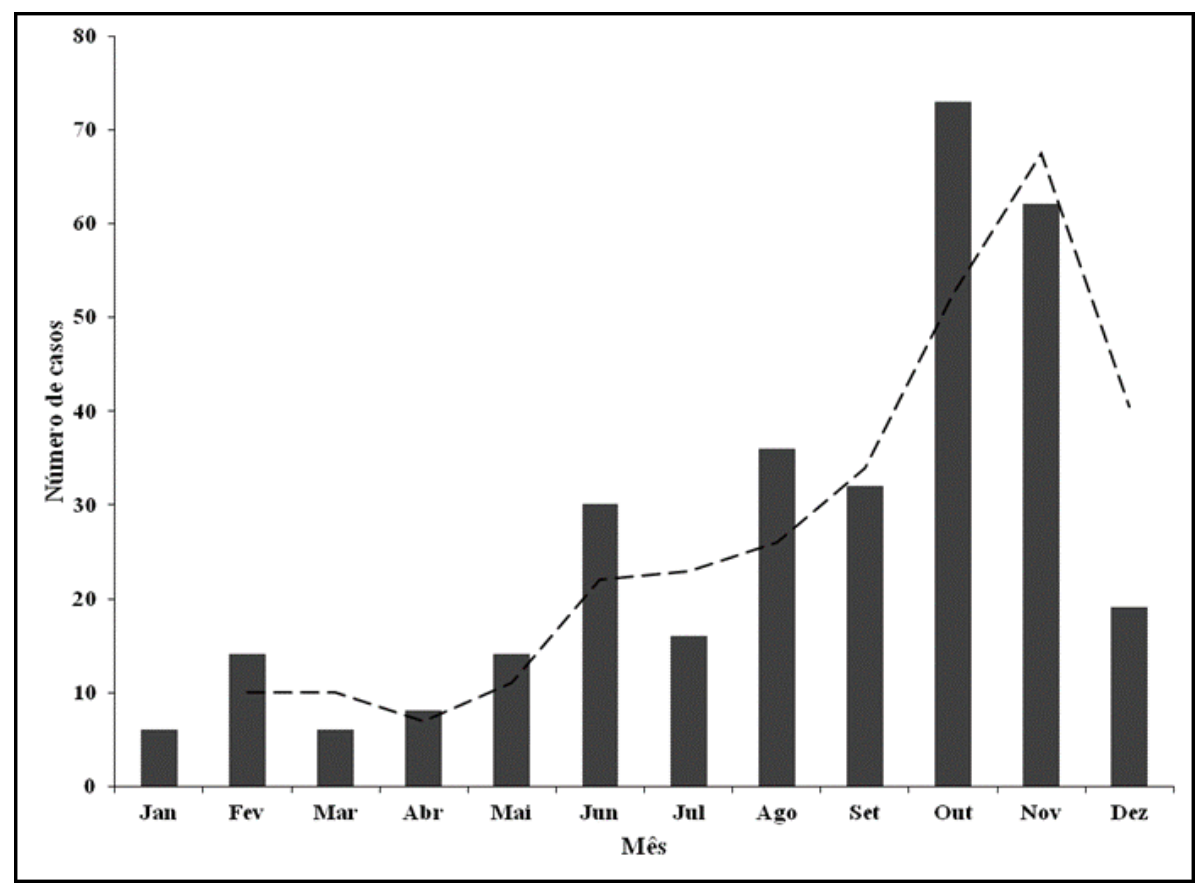

Fonte: SINAN/DATASUS.

Indivíduos do sexo masculino representaram a maior proporção dos casos $\left(\mathrm{n}=164 ; 51,9 \%\left[\mathrm{IC}_{95 \%}=\right.\right.$ 46,4\% - 57,33\%]); no entanto, esse percentual não foi discrepante em relação ao sexo feminino $(\mathrm{n}=152$; $48,1 \%\left[\mathrm{IC}_{95 \%}=42,65 \%-53,6 \%\right]$ ), corroborando com os resultados em outros estudos (BRASIL, 2015).

No que diz respeito à idade dos indivíduos, a maior incidência da doença foi observada em indivíduos adultos com idade entre 20 a 59 anos (Figura 3).

Os dados sobre escolaridade mostram que a doença acometeu mais indivíduos com baixa ou nenhuma escolaridade, que no presente estudo, totalizaram 153 indivíduos (47,37\%; $\mathrm{IC}_{95 \%}=41,99 \%-52,81 \%$ ), (Tabela 1).

As doenças infecciosas negligenciadas, como a de Chagas, estão relacionadas à pobreza em escala mundial (ORGANIZAÇÃO PAN-AMERICANA DA SAÚDE, 2016). Tal pobreza está, em muitos casos, associada à baixa escolaridade.

Dois óbitos pelo agravo foram notificados no período avaliado, que corresponde a 0,06\% dos casos. Importante ressaltar que os óbitos só levam em consideração os casos agudos, pois a fase crônica pode desencadear outros comprometimentos de saúde (BRASIL, 2009).

Na comparação dos casos confirmados por zona de residência, os dados não foram discrepantes entre o número de casos registrados na zona urbana $\left(\mathrm{n}=167 ; 52,85 \%\left[\mathrm{IC}_{95 \%}=47,34 \%-58,29 \%\right]\right)$ e na zona rural $\left(\mathrm{n}=138 ; 43,67 \%\left[\mathrm{IC}_{95 \%}=38,31 \%-49,18 \%\right]\right)$. Dois casos eram oriundos da zona periurbana (interface entre as zonas urbana e rural) e em nove casos essa informação não foi detalhada e foram categorizados como "Ignorado/Branco". 
Figura 3 - Consolidado do número de casos de DCA por faixa etária, no período de 2007 a 2017, no município de Abaetetuba, estado do Pará

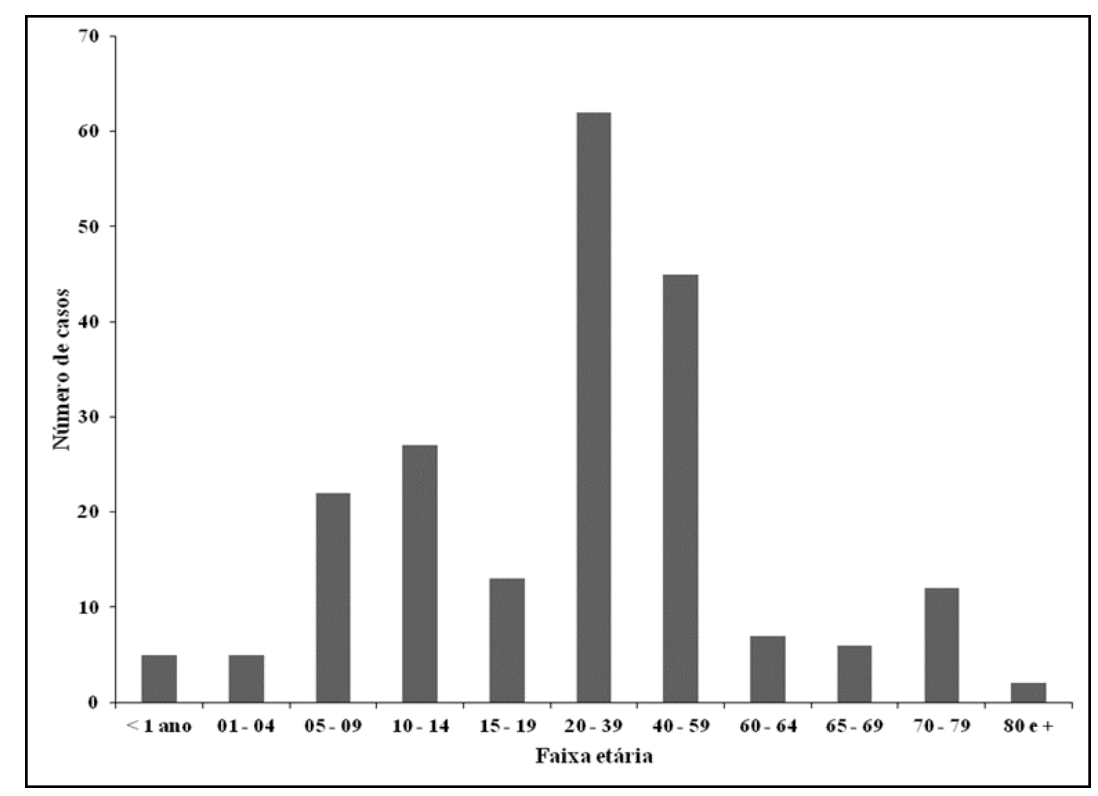

Fonte: SINAN/DATASUS.

Tabela 1 - Perfil educacional dos pacientes acometidos pela doença de Chagas aguda (DCA) no município de Abaetetuba, estado do Pará, entre 2007 a 2017.

\begin{tabular}{|c|c|c|c|c|c|c|c|c|c|c|c|c|}
\hline \multirow{2}{*}{ Escolaridade } & \multicolumn{11}{|c|}{ Ano } & \multirow{2}{*}{ Total (\%) } \\
\hline & 2007 & 2008 & 2009 & 2010 & 2011 & 2012 & 2013 & 2014 & 2015 & 2016 & 2017 & \\
\hline Ignorado/Branco & 2 & 0 & 12 & 2 & 1 & 4 & 0 & 4 & 0 & 0 & 1 & $26(8,05)$ \\
\hline Analfabeto & 0 & 2 & 1 & 1 & 0 & 2 & 0 & 1 & 0 & 1 & 1 & $9(2,79)$ \\
\hline $\begin{array}{l}1^{\text {a }} \text { a } 4^{\mathrm{a}} \text { série } \\
\text { incompleta do EF }\end{array}$ & 2 & 6 & 11 & 7 & 2 & 15 & 5 & 8 & 0 & 2 & 1 & $59(18,27)$ \\
\hline $\begin{array}{l}4^{\text {a }} \text { série completa } \\
\text { do EF }\end{array}$ & 0 & 0 & 2 & 1 & 3 & 1 & 1 & 2 & 1 & 7 & 6 & $24(7,43)$ \\
\hline $\begin{array}{l}5^{\mathrm{a}} \text { a } 8^{\mathrm{a}} \text { série } \\
\text { incompleta do } \mathrm{EF}\end{array}$ & 1 & 1 & 8 & 1 & 2 & 9 & 2 & 2 & 1 & 3 & 4 & $34(10,53)$ \\
\hline $\begin{array}{l}\text { Ensino } \\
\text { fundamental } \\
\text { completo }\end{array}$ & 0 & 0 & 3 & 0 & 0 & 0 & 1 & 1 & 10 & 6 & 6 & $27(8,36)$ \\
\hline $\begin{array}{l}\text { Ensino médio } \\
\text { incompleto }\end{array}$ & 1 & 1 & 6 & 3 & 0 & 4 & 1 & 2 & 3 & 1 & 1 & $23(7,12)$ \\
\hline $\begin{array}{l}\text { Ensino médio } \\
\text { completo }\end{array}$ & 1 & 2 & 6 & 1 & 1 & 20 & 4 & 5 & 1 & 10 & 13 & $64(19,81)$ \\
\hline $\begin{array}{l}\text { Educação superior } \\
\text { incompleta }\end{array}$ & 0 & 0 & 5 & 1 & 0 & 2 & 1 & 0 & 1 & 2 & 1 & $13(4,02)$ \\
\hline $\begin{array}{l}\text { Educação superior } \\
\text { completa }\end{array}$ & 1 & 0 & 2 & 0 & 0 & 0 & 0 & 1 & 1 & 1 & 1 & $7(2,17)$ \\
\hline Não se aplica & 2 & 2 & 4 & 0 & 0 & 7 & 3 & 8 & 3 & 1 & 7 & $37(11,46)$ \\
\hline Total & 10 & 14 & 60 & 17 & 9 & 64 & 18 & 34 & 21 & 34 & 42 & $323(100)$ \\
\hline
\end{tabular}

Fonte: SINAN/DATASUS, SESPA e Prefeitura de Abaetetuba. 
No que diz respeito ao modo de infecção, dos 316 casos confirmados, $232\left(73,42 \%\right.$; $\left[\mathrm{IC}_{95 \%}=68,29 \%-\right.$ $77,99 \%])$ estiveram associados à via oral, 20 (6,33\%) à via vetorial, um (0,32\%) à via vertical e 63 $(19,94 \%)$ foram categorizados como "Ignorado/Branco". A transmissão pela via oral é considerada como mecanismo primário, sobretudo, no ciclo silvestre (DIAS; AMATO, 2009). No ciclo enzoótico a contaminação de mamíferos susceptíveis se dá por meio da ingestão de vetores e reservatórios infectados (DIAS; COURA, 1997) .Em humanos, a transmissão do T. cruzi é esporádica e circunstancial, por meio de alimentos contaminados com triatomíneos ou suas fezes, contendo formas infectantes do protozoário, e os primeiros casos registrados ocorreram na década de 60 (DIAS, et al., 2016).

Quanto ao critério de confirmação dos casos, a quase totalidade foi determinada por meio de diagnóstico laboratorial ( $\mathrm{n}=306)$, sete pelo critério cliníco-epidemiológico, dois foram classificados como "Ignorado/ Branco" e um ainda está em investigação. Estes resultados estão em consonância com as recomendações descritas no II Consenso Brasileiro em Doença de Chagas (DIAS, et al., 2016), no qual se preconiza que os casos de DCA devem ser confirmados por meio de diagnóstico laboratorial e que o critério clínico-epidemiológico deve ser adotado apenas em casos suspeitos, com exame parasitológico negativo e sorológico inicialmente não reagente, e que possuam vínculo epidemiológico com casos confirmados de DCA por critério laboratorial, durante surto de doença na fase aguda por transmissão oral.

No que diz respeito à taxa de incidência mensal da infecção chagásica, nos meses do segundo semestre de cada ano (julho a dezembro) foram registradas as maiores incidências da doença, com destaque para o mês de novembro, conforme indicado na Figura 4.

Figura 4 - Incidência mensal de casos de doença de Chagas no município de Abaetetuba, de 2007 a 2017.

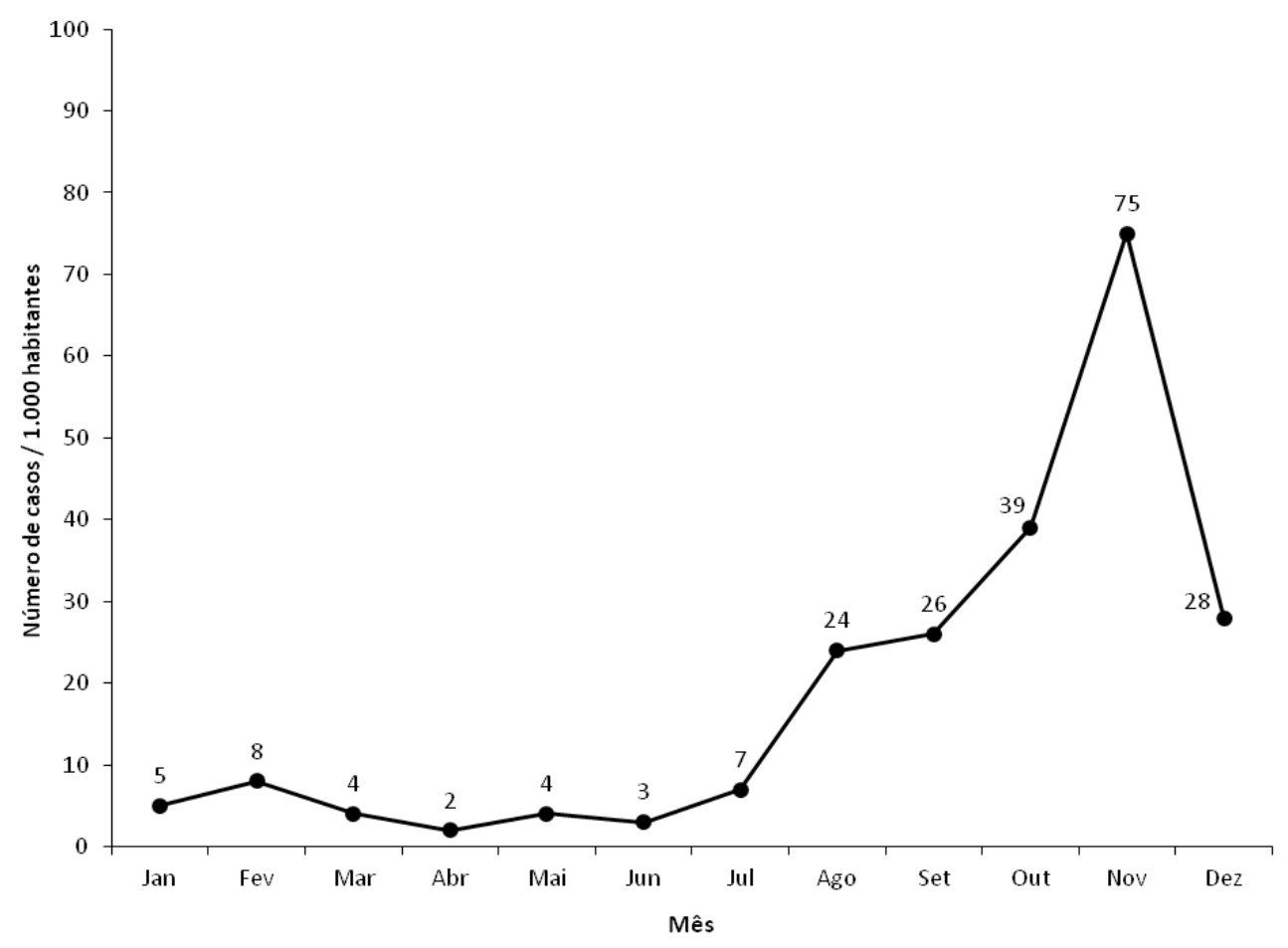

Fonte: SINAN/DATASUS, SESPA e Prefeitura de Abaetetuba.

Nos meses do primeiro semestre (janeiro a junho) há uma discreta variação no número de casos 
(2 a 8), enquanto que nos meses do segundo semestre essa variação foi visivelmente maior (7 a 75). A elevada variação na taxa de incidência na segunda metade dos anos coincide com os meses de safra do açaí na região que, contaminado pelo T. cruzi e mal manuseado durante seu preparo, determina a elevada incidência da doença por contaminação pela via oral (SOUSA JUNIOR, et al., 2017). Em vista desta problemática, o governo do estado Pará, por meio do decreto no 2.475 , artigo 135, inciso V, de 10 de setembro de 2010, estabeleceu o termo de compromisso de ajustamento de conduta entre o Ministério Público do Estado e os batedores de açaí, com o propósito de melhorias no manuseio, no preparo e na qualidade do produto (PARÁ, 2010). Os moradores da zona urbana do município manipulam a produção do seu açaí na própria residência, e a falta de campanhas informativas para essa população contribui para o alto índice da contaminação pela via oral. A limitação do estudo esteve em não estabelecer a forma de contaminação predominante da zona rural e urbana do município.

\section{Conclusão}

Os dados apontados no presente estudo reforçam o status de endemicidade da DCA no estado do Pará. O elevado número de casos confirmados em Abaetetuba no período avaliado (326 em 10 anos) é uma realidade presente também em outros municípios, sobretudo naqueles situados na região Nordeste do estado.

O aumento das notificações de DCA na segunda metade do ano, coincidente com o período da safra do açaí - alimento que faz parte da dieta da maioria da população - mostra que a transmissão oral é o principal mecanismo de transmissão da doença para humanos no município.

A alta prevalência da infecção chagásica em indivíduos com idade entre 20 e 59 anos com baixa ou nenhuma escolaridade, de áreas rural e urbana, indica a necessidade de campanhas de educação em saúde constantes voltadas para este público-alvo, no sentido de alertar sobre o principal mecanismo de transmissão (via oral) e as formas de se evitar a contaminação por essa e por outras vias. Adicionalmente, o diagnóstico da DCA deve ser ainda mais oportuno na rede básica de saúde municipal, com vistas ao correto manejo dos casos. O medicamento indicado para o tratamento (Benznidazol) deve ser fornecido aos pacientes gratuitamente.

Por fim, a capacitação dos batedores de açaí, no que se refere às boas práticas na manipulação e higiene dos frutos, deve ser frequente e continuada, ao mesmo tempo em que a fiscalização dos locais de revenda do produto deve ser intensificada. A adoção em conjunto dessas medidas, poderia ter um efeito realmente preventivo na transmissão da DCA no município e no Estado.

\section{REFERÊNCIAS}

BERN, C. et al., Trypanosoma cruzi and Chagas disease. In:The United States Clin Microbiol Rev 2011,v.24, n.4, p.655-81.

BRASIL. MINISTÉRIO DA SAÚDE. SINAN. Dados Complementares do Caso. Ficha de investigação doença de chagas aguda sistema de informação de agravos de notificação, 2009. Disponível em: <http:// tabnet.datasus.gov.br/cgi/tabcgi.exe?sinannet/cnv/chagasbr.def $>$.

BRASIL. MINISTÉRIO DA SAÚDE. Secretaria de Vigilância em Saúde. Doença de Chagas aguda no Brasil: série histórica de 2000 a 2013. Bol. Epidemiol.v. 46, n.21, p 1-9, 2015.

CHAGAS, C. Nova tripanozomiase humana: estudos sobre a morfolojia e o ciclo evolutivo do Schi- 
zotrypanum cruzi n. gen., n. sp., ajente etiolojico de nova entidade morbida do homem. Memórias do Instituto Oswaldo Cruz, Rio de Janeiro, v.1, n.2, p.159-218. 1909d.

COURA, J. R. Síntese das doenças infecciosas e parasitárias. Rio de Janeiro:Guanabara Koogan,2008. p. 314.

DIAS, J. C. P.; AMATO, N.V. Prevenção referente às modalidades alternativas de transmissão do Trypanosoma cruzi no Brasil. Rev Soc Bras Med Trop. v.2, p. 68-72, supl. 44, 2011.

DIAS, J. C. P.; COURA, J. R. org. Clínica e terapêutica da doença de Chagas: uma abordagem prática para o clínico geral [online]. Rio de Janeiro: Fiocruz, 1997.

DIAS, J.C.P.; RAMOS Jr, A.N.; CORREIA, D. Consensus on Chagas Disease, 2015. Rev Soc Bras Med Trop, v. 4, supll.1, 2016.

FERREIRA, L.R. et al. Interferon- $\gamma$ and other inflammatory mediators in cardiomyocyte signaling during Chagas disease cardiomyopathy. World J Cardiol. v.6, n. 8, p. 782-90, 2014.

GASCON, J.; BERN, C.; PINAZO, M-J. Chagas disease. In: Spain, the United States and other non-endemic countries. Acta Trop, v. 115, n.1-2, p.22-7, 2010.

HOTEZ, P.J.; FUJIWARA, R.T. Brazil's neglected tropical diseases: an overview and a report card. Microbes Infect. v.16, n.8, p. 601-6, 2014.

INSTITUTO BRASILEIRO DE GEOGRAFIA E ESTATÍSTICA (IBGE). Cidades, Abaetetuba-Pa. Disponível em: <https://cidades.ibge.gov.br/brasil/pa/abaetetuba/panorama $>$.

SOUSA JUNIOR, A. da .S.; et al.; Análise espaço-temporal da doença de Chagas e seus fatores de risco ambientais e demográficos no município de Barcarena, Pará, Brasil. Rev. Bras. Epidemiol.v.20, n. 4, p. 742-755, Out-dez 2017.

MARQUES, J. As manifestações de ECG do maior surto da doença de Chagas devido a infecção oral na América Latina. Arquivos Brasileiros de Cardiologia. v. 101, n. 3, p. 249-254, 2013. DOI 10.5935/ abc. 20130144 .

MARTINS-MELO, F. R.; RAMOS Jr, A.N., ALENCAR, C.H. HEUKELBACH J. Prevalence of Chagas disease. In: Brazil: A systematic review and meta-analysis. Acta Trop. v.30, p.167-174, 2014.

BRASIL. MINISTÉRIO DA SAÚDE. Secretaria de Vigilância em Saúde. Doença de Chagas aguda no Brasil: série histórica de 2000 a 2013. Bol. Epidemiol.v. 46, n.21, p 1-9, 2015.

MONCAYO, Á., SILVEIRA, A. C. Current epidemiological trends for Chagas disease in Latin America and future challenges in epidemiology, surveillance and health policy. Mem Inst. Oswaldo Cruz, Rio de Janeiro, v.l. 104 (Suppl. I), p. 17-30, 2009.

MONTEIRO, W.M. et al., Trypanosoma cruzi I and IV stocks from Brazilian Amazon are divergent in 
terms of biological and medical properties in mice. PLoS Negl Trop Dis. v. 7, n.2, p. e2069, 2013.

ORGANIZAÇÃO PAN-AMERICANA DA SAÚDE. 55 Conselho Diretor. 68 $^{\text {a }}$ Sessão do Comitê Regional da OMS para as Américas. Washington, D.C., EUA, 26-30 setembro de 2016.

PARÁ. Decreto no 2.475, de 13 de setembro de 2010. Diário oficial do estado do Pará no 31.750. 04 Cadernos 64 Páginas. Disponível em <http://www.ioepa.com.br/pages/2010/09/13/2010.09.13.DOE_5.pdf.

PINTO, A.Y. et al.; Dupla abordagem de infecções por Trypanosoma cruzi e/ou Plasmodium spp. aplicadas ao diagnóstico de doença de Chagas e exposição vetorial triatomínica na Amazônia brasileira. Rev Pan-Amaz Saúde,v. 6, n. 1, p. 35-43, 2015.

PINTO, A.Y.N. Fase aguda da doença de Chagas na Amazônia brasileira: estudo de 233 casos do Pará, Amapá e Maranhão observados entre 1988 e 2005. Rev Soc Bras Med Trop. v. 41, n.6, p. 602-14, nov.dec.2008.

RODRIGUES, R.P.S. Características epidemiológicas, zoonóticas, clínicas, patológicas e diagnósticas da doença de Chagas. PUBVET, v.10, n.3, p.200-206, mar.2016.

SANTOS, R.S.; COELHO-FERREIRA, M. Estudo etnobotânico de Mauritia flexuosa L. f. (Arecaceae) em comunidades ribeirinhas do Município de Abaetetuba, Pará, Brasil. Acta amazônica. v.42, n.1, p.1$10,2012$.

SINAN/DATASUS http://tabnet.datasus.gov.br/cgi/tabcgi.exe?sinannet/cnv/chagasbr.def, acesso em: 14/09/2018). Disponível em: http://datasus.saude.gov.br/.

SOUSA JUNIOR, A. da .S.; et al.; Análise espaço-temporal da doença de Chagas e seus fatores de risco ambientais e demográficos no município de Barcarena, Pará, Brasil. Rev. Bras. Epidemiol.v.20, n. 4, p. 742-755, Out-dez 2017.

SOUZA, C.N.P.; et al. Fatores contribuintes à ocorrência de mortalidade por doença de chagas. Rev. Bras. Biom., São Paulo, v. 32, n. 4, p.544-552, 2014.

WORLD HEALTH ORGANIZATION. Chagas disease (American trypanosomiasis) [Internet]. Geneva: World Health Organization; 2015 [citado 2015 Nov 13]. Disponível em: http://www.who.int/mediacentre/ factsheets/fs340/. 